\title{
LIBERTÉ DE PRESSE ET SECRET JUDICIAIRE : POUR UNE RÉGULATION PARTAGÉE
}

\section{Philippe Gerday ${ }^{1}$}

Outil juridique indispensable au journaliste, la liberté de presse est au fondement des démocraties modernes. Ces dernières assignent aux médias la mission d'informer le public sur tout ce qui relève du fonctionnement de la société. Le développement des affaires judiciaires comme l'état de santé général de la justice font partie du champ naturel d'investigation de la presse.

Cette liberté d'enquêter, de s'exprimer et de publier n'est toutefois pas absolue. Elle entre en concurrence avec d'autres libertés et droits fondamentaux en vertu desquels elle connaît des restrictions. C'est tout particulièrement vrai dans le domaine judiciaire, où le secret institutionnalisé veille au respect des personnes (présomption d'innocence, honorabilité, vie privée) et à la bonne fin des devoirs de justice.

Qui doit assurer l'équilibre entre ce droit de la justice au secret et le droit du public de savoir? Qui doit sanctionner les débordements ?

1 Journaliste au quotidien Vers l'Avenir, Diplômé d'études approfondies en communication de l'UCL.

Recherches en communication, $\mathrm{n}^{\circ}$ 9, (1998). 
Juges et journalistes s'en disputent l'arbitrage. Droit et déontologie s'affrontent, au nom du public, du justiciable, de l'intérêt général. D'un côté, parquets, cours et tribunaux -de l'extérieur des médiasrevendiquent la régulation finale du droit d'informer. De l'autre, les praticiens de la presse -de l'intérieur cette fois- érigent l'autocontrôle comme seule régulation opportune de la liberté d'enquêter et de rendre public.

La présente contribution soutient que loin de s'exclure, ces deux régulations -juridique et déontologique-se complètent. Éditeurs et journalistes ont pour eux l'intelligence du métier mais ils ignorent la sanction dissuasive. Les praticiens de la justice en revanche, s'ils n'ont pour eux que le regard externe sur la presse, disposent des moyens de contrainte faisant défaut à l'autocontrôle médiatique.

Les lignes qui suivent tentent de montrer en quoi la mixité des contrôles est le dispositif le plus efficace pour réguler liberté d'information et secret judiciaire. Faire se rencontrer juges et journalistes en un même lieu, pour confronter valeurs et logiques en œuvre dans les médias et l'institution judiciaire, doit être la meilleure voie, non pour neutraliser liberté de presse et État de droit, mais pour les renforcer conjointement.

\section{L'empire du milieu}

Si la liberté de presse avait valeur de "primus inter pares" au sein des droits constitutionnellement reconnus, le problème du contrôle ne se poserait pas. Rien ne ferait en principe barrage à la liberté d'enquêter, de s'exprimer et de publier qui, telle un phare dans la nuit, guiderait souverainement les arpenteurs du vivant dans les méandres du quotidien.

On n'en est pas là. Composante fondamentale des démocraties modernes, la liberté d'informer n'est qu'un des droits dont disposent les Belges et la plupart des Occidentaux. Il y en a d'autres, comme le droit à la vie privée ou le droit à la présomption d'innocence (avant toute sanction définitive d'un tribunal), pour s'en tenir au registre judiciaire.

Ces droits ont un poids juridique équivalent. Ils sont sur pied d'égalité, dans la Constitution belge -puisque c'est le système belge, comparable toutefois à de nombreux systèmes voisins, qui est pris ici pour référence- et d'autres instruments juridiques comme la 
Convention européenne de sauvegarde des droits de l'homme et des libertés fondamentales. Aucune hiérarchie prétablie entre ces principes : quand ils entrent en concurrence, il faut à chaque fois pondérer en fonction des circonstances et des intérêts en présence. La subordination automatique d'un de ces droits à un autre ne joue pas.

C'est éminemment le cas quand la liberté de presse entre en conflit avec le droit à la vie privée ou le droit à un procès équitable ${ }^{1}$. Le secret judiciaire résiste aux investigations du journaliste, pour le bien des enquêtes en cours ou la réputation des parties à la cause (prévenus, victimes ou témoins). Mais ce droit de la justice au secret n'est pas absolu. Comme la liberté de presse, il souffre de restrictions. Quand, pour en rester à des exemples forts, une personnalité de premier plan entre dans le collimateur de la justice, quand des enquêteurs recourent à des procédés inavouables, quand des témoins tentent de dévoyer une instruction ou des magistrats font montre d'un attentisme suspect, le public a le droit de savoir, donc le journaliste, le devoir de le dire.

Mais en dehors de ces situations tranchées, l'équilibre raisonnable entre le droit du public de savoir et le droit de la justice au secret n'est pas aisé à trouver. C'est un équilibre en permanence instable. Il n'y a pas de recette pour le juste milieu. Sur ce point, les grands textes fondateurs sont muets, laissant libre cours aux interprétations les plus diverses.

L'unanimité est remarquable quand il s'agit de fonder la liberté de presse, celle d'investiguer, de s'exprimer et de publier sur tout ce qui relève de l'intérêt public :

"La liberté de manifester ses opinions en toute manière" est garantie, "la presse est libre " et " la censure ne pourra jamais être établie" (articles 19 et 25 de la Constitution belge) ;

"Tout individu a droit à la liberté d'opinion et d'expression, ce qui implique le droit de ne pas être inquiété pour ses opinions et celui de chercher, de recevoir et de répandre, sans considération de frontière, les informations et les idées par quelque moyen que ce soit" (article 19 de la Déclaration

1 Voir J. VeLu, Propos sur les normes européennes applicables aux relations entre la justice et la presse, traduction française du discours prononcé à l'audience solennelle de rentrée de la Cour de cassation, Bruxelles, le ler septembre 1995, texte dactylographié, p. 21-26. Voir aussi G. DE LEVAL, La justice civile et la presse, in Coordonnées du colloque "Justice et médias", Bruxelles, décembre 1995, p. 117-119. 
universelle des droits de l'homme - Moniteur belge du 31 mars 1949) ;

“Toute personne a droit à la liberté d'expression. Ce droit comprend la liberté d'opinion et la liberté de recevoir ou de communiquer des informations ou des idées sans qu'il puisse y avoir ingérence d'autorités publiques et sans considération de frontière" (article 10 paragraphe 1er de la Convention européenne de sauvegarde des droits de l'homme et des libertés fondamentales, approuvée par la loi belge du 13 mai 1955 Moniteur du 19 août 1955);

“ Toute personne a droit à la liberté d'expression ; ce droit comprend la liberté de rechercher, de recevoir et de répandre des informations et des idées de toute espèce, sans considération de frontière, sous une forme orale, écrite, imprimée ou artistique, ou par tout autre moyen de son choix " (article 19 paragraphe 2 du Pacte international relatif aux droits civils et politiques, approuvé par la loi belge du 15 mai 1981 Moniteur du 6 juillet 1983) ${ }^{1}$.

L'institution judiciaire n'est pas épargnée par ce droit de savoir et de se former une opinion. Les journalistes ont à rendre compte du travail de la justice, de la manière dont elle s'organise jusqu'au traitement du moindre dossier. Le public s'attend à trouver, dans la presse, un bulletin de santé de l'institution judiciaire, son état de forme comme ses défaillances, structurelles ou passagères :

Les gouvernements des pays membres du Conseil de l'Europe, dans leur résolution praguoise de décembre 1994 sur les libertés journalistiques et les droits de l'homme, rappelaient que "le maintien et le développement d'une démocratie véritable exigent l'existence et le renforcement d'un journalisme libre, indépendant, pluraliste et responsable. Cette exigence se traduit par la nécessité pour le journalisme d'informer les individus sur l'activité des pouvoirs publics et du secteur privé, en leur donnant ainsi la possibilité de se former une opinion (...) et de soumettre l'exercice des divers pouvoirs à un continuel examen critique" 2 . Or la justice fait partie de ces divers pouvoirs.

1 Pour ces textes fondamentaux, voir S.-P. DE Coster et Fr. JongEN, Médialex 1997. Recueil de textes commentés, Kluwer, Diegem, 1996, p. 5-26.

2 Conférence ministérielle du Conseil de l'Europe, Les media dans une société démocratique. Déclaration politique, résolutions et communication, Prague, 7-8 décembre 1994, p. 12-13. 
L'ancien procureur général près la Cour de cassation belge, Jacques Velu, estimait pour sa part que " non seulement les citoyens sont libres de leurs opinions sur les affaires judiciaires et sur le fonctionnement de la justice, mais la presse a le droit de leur communiquer en la matière des informations et des idées même dérangeantes et ils ont aussi le droit de recevoir de la presse ces informations et ces idées. En ce domaine, comme dans d'autres, la presse doit pouvoir jouer, suivant la formule consacrée, son rôle indispensable de «chien de garde»"1.

Cette liberté de "chercher à savoir" et d'informer, on l'a dit, n'est pas sans contraintes. En matière judiciaire notamment, elle est soumise à restrictions dans un nombre limité de cas :

L'article 10 de la Convention européenne des droits de l'homme, qui en son paragraphe premier reconnaît à toute personne le droit à la liberté d'expression, d'opinion et d'information, précise en son second paragraphe que "l'exercice de ces libertés comportant des devoirs et des responsabilités peut être soumis à certaines formalités, conditions, restrictions ou sanctions, prévues par la loi". Ces mesures, dans une société démocratique, sont estimées nécessaires à la sauvegarde de certaines valeurs dont "la défense de l'ordre et la prévention du crime (...), la protection de la réputation ou des droits d'autrui, pour empêcher la divulgation d'informations confidentielles ", ou la garantie de "l'autorité et l'impartialité du pouvoir judiciaire".

La jurisprudence de la Cour européenne des droits de l'homme donne des contours plus précis à cet interdit partiel ou temporaire d'informer. En matière de justice, les ingérences légales dans l'exercice de la liberté de presse visent à protéger le pouvoir judiciaire d'articles ou de reportages jugeant prématurément, exposant les parties en présence à des dommages ou à des pressions, ou portant atteinte au secret d'une enquête et à sa bonne marche? .

En droit belge, ces ingérences légales prennent la forme de mesures générales (assurer à chaque justiciable le respect de la présomption d'innocence, le droit à un procès équitable ou la sauvegarde de la vie privée et familiale) ou particulières (protéger les mineurs d'âge, les victimes de viol et d'abus sexuels, ou les personnes impliquées dans une procédure de

1 J. VELU, op. cit., p. 9.

2 Ibid., p. 17. 
divorce par l'interdiction de publier leurs noms ou tout autre élément d'identification).

La déontologie journalistique elle-même bannit certaines pratiques ou attitudes, recoupant les interdits légaux. Dans le code belge commun aux journalistes et aux éditeurs (ABEJ-FNHI-AGJPB), qui applique les grands principes déontologiques des déclarations de Bordeaux (1954) et de Munich (1971), on trouve notamment les règles suivantes ${ }^{1}$ :

La probité au service de la vérité (aucune méthode déloyale ou malhonnête pour obtenir informations, photos ou documents ; ne cacher aucune information essentielle; ne rien falsifier; rectifier après coup toute information inexacte ou nuisible) ;

Le respect des personnes et de leur vie privée (pas d'intrusion illicite dans l'intimité ou la peine des gens... à moins que l'intérêt de la société ou de la liberté de la presse ne l'exige).

Comme on va le voir, cette remarque restreignant l'intimité des gens vaut son pesant d'or car elle est au centre du débat qui nous occupe. Les grands principes sont fixés, tant par le droit que par la déontologie. Mais quid de leur application? Qui va baliser le droit chemin, et qui surtout sanctionnera les écarts ? C'est aux parquets, cours et tribunaux à trancher, soutiennent juristes et magistrats. Non, rétorquent les praticiens de la presse : c'est à la juridiction de ses pairs que doit être soumis tout journaliste, "à l'exclusion de toute ingérence gouvernementale ou autre ", souligne la déclaration de Munich. On est en pleine guerre de tranchées...

\section{L'équilibre de la terreur}

D'un côté, la plupart des juges estiment que la loi, qui vaut pour tous, les a idéalement placés, au-dessus de la mêlée, pour à la fois sauvegarder l'intérêt général et rendre effective la responsabilité de la

1 Voir D. VooRHoor, "De relatie media en justitie vanuit het perspectief van de persvrijheid en de vrijheid van expressie en informatie", in Justice et médias. Trois avis préliminaires à la demande du ministre de la Justice, partie C, Bruxelles, 1995, p. 38-40. Voir aussi l'Aide-mémoire de la presse judiciaire, édité par l'AGJPB, p. 3-5. 
presse, moyennant sanctions. Les journalistes ne peuvent se prévaloir, à ce titre, d'aucune immunité.

De l'autre, la plupart des journalistes se proclament " juges naturels " des pratiques de presse, seuls véritablement habilités à préserver la liberté d'expression, fondement de toute démocratie. Parquets, cours et tribunaux ne sont pas vus comme des juges "illégitimes" des médias (seule une minorité extrême va jusque-là), mais comme des juges "inopportuns", surtout en matière judiciaire. Pour réguler la liberté de presse, les praticiens des médias revendiquent un authentique... privilège de juridiction.

Et chacun a bien entendu ses armes pour faire valoir son point de vue. Si la presse se fait trop remuante ou vindicative, les codes pénal et civil ne manquent pas de ressources pour pacifier les esprits (voir ci-dessous). Si les juges par contre semblent en faire trop, une campagne de presse ou quelque article incendiaire auront tôt fait de calmer les menaces, rien ne faisant " tant saigner qu' une coupure de presse ", comme le dit Audouard.

Au cours du colloque organisé au Sénat belge sur la justice et les médias, en décembre 1995, le procureur du Roi de Bruxelles, Benoît Dejemeppe, décrivait la situation en ces termes :

Le traitement des dossiers les plus graves se déroule selon un scénario prévisible : aux dérapages émotifs, sensationnalistes, pour tout dire légers de certains médias, succèdent des enquêtes puis les protestations courroucées de la presse redoutant quelques atteintes obliques à la liberté d'expression. Chaque camp se trouve pétrifié par une espèce d'équilibre de la terreur. L'armistice est alors promptement décrété, dans la confusion. Quant à la véritable question posée, à savoir le partage des responsabilités entre le journaliste, le législateur et le juge, elle est renvoyée aux calendes grecques. Ou au prochain dérapage. Ponce-Pilate n'est pas loin. Le spectacle est terminé, circulez, il n'y a plus rien à voir. On recommencera demain ${ }^{1}$.

Sur quoi repose la dissuasion juridique? Sur un arsenal de mesures qu'on peut rappeler ici :

- le délit de presse, ou la manifestation délictueuse d'une opinion par voie imprimée (passible des assises en Belgique). Même si les parquets ne poursuivent quasiment plus, en raison de leur

1 B. DEJEMEPPE, "Indécisions judiciaires, urgences législatives", in Coordonnées $d u$ colloque "Justice et médias", op. cit., p. 247-249. 
engorgement et de la lourdeur procédurière des procès d'assises, le délit de presse - que plusieurs propositions de loi veulent réformer reste la clé de voûte du système répressif en Belgique ;

- le recel, ou la détention illicite d'informations ou de documents "volés" ou "détournés". Au sens strict de l'article $505 \mathrm{du}$ code pénal belge, le journaliste est receleur dès qu'il détient une information ou une pièce qu'il sait avoir été soustraite à son légitime propriétaire (le ministère de la Justice, par exemple, pour un procès verbal ou un élément de procédure), en dehors même de toute publication. Une jurisprudence moins sévère estime toutefois que la simple copie d'un procès-verbal ne suffit pas à qualifier le recel ;

- la complicité de viol du secret professionnel, quand un journaliste est suspecté d'avoir poussé à la faute un fonctionnaire, un enquêteur ou un magistrat tenus au secret (article 458 du code pénal belge). En plus du risque de recel, ce journaliste peut être poursuivi pour avoir contribué, d'une manière ou d'une autre (pressions, chantage, corruption...), au viol du secret ;

- la procédure en référé, lorsque le juge est saisi en urgence d'une demande d'interdiction de publication ou de diffusion parce qu'atteinte serait portée, par exemple, à la réputation d'autrui, à sa vie privée ou à sa présomption d'innocence. Même si le succès de ces recours semble limité (la plupart des juges estimant que ce genre d'actions porte atteinte aux principes constitutionnels de liberté de presse et d'interdiction de la censure), le référé arrive parfois à perturber l'activité des périodiques d'information ou des magazines télévisés ;

- le recours devant le juge civil, sur base des articles 1382 à 1384 du code civil belge traitant des "quasi-délits". Ces articles prévoient la réparation de tout dommage par celui qui l'a causé, en dehors même de toute faute pénale. Si le dommage est établi, et un lien fait avec la publication incriminée, les journalistes et entreprises de presse peuvent être contraints à s'acquitter de réparations sonnantes et trébuchantes qui atteignent parfois le "punitif", pour un préjudice porté à l'intimité, l'honneur ou la réputation de personnes ou de sociétés.

Même si ne sont pas rares les magistrats (ministère public comme juge de fond) refusant d'appliquer une quelconque de ces mesures dès qu'il s'agit de la presse, l'équilibre de la terreur ne fonctionne pas toujours. Des journalistes sont " condamnés ", au pénal comme au civil, ce qui, en dehors des cas flagrants de brebis galeuses, 
contribue à alimenter le ressentiment du quatrième pouvoir envers la justice.

D'autant, on l'a dit, que les praticiens de la presse s'estiment seuls maîtres à bord -ou quasi-quand il s'agit de juger l'orthopraxie des confrères. Le code de principes de journalisme en vigueur à l'AGJPB (association générale des journalistes professionnels de Belgique) prévoit l'autocontrôle déontologique comme enceinte de jugement des actes journalistiques.

Les journalistes établiront la licéité des intrusions dans l'intimité ou la peine des gens, en fonction de "l'intérêt de la société ou de la liberté de presse" (voir ci-dessus). Mieux : si une opposition apparaît entre le droit à la libre expression et un autre droit de l'homme fondamental, c'est à l'éditeur ou au rédacteur en chef, après avoir consulté les journalistes concernés, que revient la responsabilité de donner la priorité à l'un de ces deux droits (point $10 \mathrm{du}$ code).

A tous ceux, magistrats ou autres, qui voudraient remédier aux erreurs et négligences journalistiques en dehors des instances de contrôle déontologique de la profession (créées en 1995 au sein de l'AGJPB), l'association des journalistes belges répond :

Nous sommes seuls juges de notre déontologie. Et même si des pressions extérieures tendant à régenter les journalistes et leurs cuvres augmentent de jour en jour, nous entendons y veiller nous-mêmes ${ }^{1}$.

Le constat est clair : deux " camps" s'opposent pour réguler la liberté de presse, au regard du secret judiciaire comme de tout autre domaine. Il y a les partisans du contrôle juridictionnel externe ou hétérorégulation des juges, attribuant aux parquets, cours et tribunaux la charge de réprimer les mésusages du droit de savoir et d'informer; et en face, les partisans du contrôle déontologique interne ou autorégulation, ne reconnaissant qu'aux praticiens de presse le droit " naturel" de juger.

\section{Statu quo bancal}

Ces relations conflictuelles ne chagrinent pas tout le monde. Juges ou journalistes, certains pensent que cet équilibre de la terreur

1 Annuaire officiel de la presse belge 1997-1998, Bruxelles, AGJPB, 1997, p. 37. 
porte ses fruits et qu'à tout prendre, il ne faut toucher à rien. Ces deux mondes de la justice et des médias sont antagonistes par nature. Il faut donc les laisser en tension permanente et en dissuasion réciproque. C'est la seule manière d'entretenir l'équilibre des forces.

D'autres au contraire, comme le procureur du Roi de Bruxelles, considèrent le statu quo bancal. C'est aussi notre point de vue, pour quatre raisons au moins.

\section{Faiblesses naturelles de la monorégulation}

Il y a les faiblesses naturelles de la monorégulation. Qu'il s'agisse de celle des journalistes ou de celle des juges, la monorégulation exclusive (autorégulation déontologique ou hétérorégulation judiciaire) pèche par défaut.

La monorégulation externe, dans le cas du contrôle judiciaire des médias, souffre des travers de la régulation prescriptive, qui produit des ordres formels et contraignants sans normes ni orientations. Baliser le travail ordinaire des journalistes n'est pas du rôle des cours et tribunaux, qui se contentent de réprimer les écarts par rapport au droit.

A l'inverse, la monorégulation interne, dans le cas de la presse, présente les carences de la régulation normative, qui ne dégage que des règles et des préceptes sans prescrits ni sanctions. Punir les débordements de journalistes ne figure pas parmi les missions actuelles des instances déontologiques de l'AGJPB, qui se contentent, saisies de plaintes, de dire ce qui aurait dû être fait ou évité dans tel et tel cas. Les journalistes jugent leurs pairs mais ne les sanctionnent pas.

L'absence de véritables organes de contrôle dotés de pouvoirs "répressifs" est d'ailleurs la difficulté majeure de la régulation interne de la presse à travers le monde. Comme le signale Daniel Cornu, c'est la tentation du "voyage sur le marchepied" de la communication, par allusion aux usagers des transports publics qui refusent de payer leur billet ${ }^{1}$.

La plupart du temps, les journalistes se réfugient derrière la protection officielle que leur offre la régulation déontologique (les déclarations de Bordeaux, de Munich, appuyées par les codes de pratiques nationaux) sans toutefois satisfaire aux obligations qu'elle

1 D. CORNu, Journalisme et vérité. Pour une éthique de l'information, Genève, Labor et Fides, 1994, p. 129. 
induit. Au regard de la société, ils sont "sous contrôle " alors qu'en réalité, ils naviguent en régime de liberté peu ou mal surveillée.

Comme les conseils de presse restent par trop cantonnés dans la seule interprétation déontologique des règles, ignorant amende, suspension ou exclusion, le public (magistrats et législateur entre autres) est tenté d'y voir un écran de fumée protecteur, mettant journalistes et éditeurs à l'abri des intrusions inamicales, donc de reprendre les choses en mains au moyen de lois plus strictes et de recours plus nombreux en justice.

Mais la régulation externe, sans une intelligence fine -de 1" intérieur" pourrait-on dire- des logiques et valeurs en présence dans l'exercice du métier de presse, court le risque de "profaner" la déontologie par des décisions trop carrées. Quand un journaliste ou un organe de presse rompent le secret de l'instruction, entravent la vie privée d'un inculpé ou malmènent l'honorabilité d'un enquêteur, leurs motifs peuvent être obscurs et condamnables (comme la recherche inconsidérée du scoop ou le règlement de comptes personnel), mais ils peuvent tout aussi bien être clairs et louables (comme la dénonciation d'une supercherie, d'une manipulation policière ou d'une cabale de juges). Il n'est pas certain dans ce cas que parquets, cours et tribunaux soient idéalement placés, toujours et partout, pour apprécier seuls la distinction des mobiles.

Ces faiblesses de la monorégulation sont à nos yeux l'argument le plus fort pour concilier, au même moment et en un même lieu, les deux formes de contrôle -judiciaire et déontologique- des pratiques de presse. Est suggérée ici une instance commune de régulation, regroupant l'interne et l'externe (les journalistes et les juges), pour guider les relations très particulières entre la presse et la justice. C'est l'option prise pour la polyrégulation.

\section{Difficulté de type axiologique}

Un autre argument de conciliation est la difficulté de type axiologique à arbitrer les conflits de valeurs entre la liberté de presse et les autres droits fondamentaux. C'est la fameuse pondération des intérêts en présence évoquée ci-dessus.

Henri Pigeat résume le problème en ces termes :

La puissance des médias fascine et inquiète. Ils apparaissent souvent comme le champ d'un jeu sans règles, sans responsabilité et sans réelle sanction. A cette impression bien 
sûr excessive, s'oppose l'aspiration naturelle de toute société à des normes simples, claires et aussi complètes que possible pour définir précisément les pouvoirs des médias, mais aussi leurs responsabilités. Là gît la difficulté. L'activité des médias repose sur le principe de la liberté d'expression qui ne se divise pas, s'encadre difficilement et s'affaiblit vite devant des limites trop rigoureuses ${ }^{1}$.

Si les limites placées à la liberté de presse sont trop rigoureuses, elles la nient. Si ces limites sont par contre trop lâches, elles menacent les autres libertés, favorisant l'usage dévergondé ou tyrannique de la liberté de presse. La fixation des limites, dans le concret des pratiques de presse, n'est pas plus aisée aux déontologues qu'aux magistrats, mais un travail concerté diminue les risques d'erreur. C'est pourquoi juges et journalistes ont intérêt à évaluer en commun.

\section{Difficulté de type épistémologique}

Le même argument de conciliation prévaut devant les difficultés de type épistémologique à tracer les contours précis de la vérité journalistique.

Daniel Cornu écrit :

Il est dans la nature de l'information de dire la vérité, et toute vérité n'est pourtant pas bonne à dire. Il existe dans le travail journalistique et particulièrement dans la recherche de l'information, une zone d'incertitude, qui oblige le journaliste à engager son propre jugement, à mettre lui-même en balance l'intérêt du public, qui seul légitime sa fonction, et la protection auxquelles les personnes ont droit. Cette zone est assez large pour que s'y installent, à propos d'un même objet, des avis divergents. Non seulement entre les journalistes et les juges. Mais aussi entre les journalistes... et même entre juges ${ }^{2}$.

Le journaliste est diseur de vérité. Il a pour fonction principale de rechercher des faits dignes d'être signalés, de les vérifier, d'en dégager le sens et de les raconter. Un travail journalistique bien fait est une somme d'objectivité dans l'observation, d'impartialité dans l'interprétation et d'authenticité dans la narration.

1 H. PIGEAT, Médias et déontologie. Règles du jeu ou jeu sans règles, Paris, PUF, 1997, p. 3.

2 D. CoRnu, op. cit., p. 445-446. 
Or cette exactitude des faits, cette justesse des jugements et cette "véridicité " des récits -horizons ultimes-, c'est beaucoup demander à un seul homme. Il y a forcément une zone d'incertitude, comme l'appelle Cornu, qui force le journaliste à confronter sa personnalité et ses attentes à celles des autres (ses sources, son public, ses confrères) pour serrer la vérité au plus près.

Par nature, cette zone est large, abritant des avis parfois très divergents. C'est pourtant dans cette zone d'incertitude que journalistes, public et juges devront trancher quant à la vérité d'une opinion, d'un article, d'une émission. C'est dans cette zone qu'ils devront apprécier l'éventuelle liberté prise avec les événements, mais aussi évaluer la justesse d'un jugement ou la véridicité d'un récit.

La vérité journalistique ne se limite pas aux faits : elle englobe également évaluations et opinions, ce qui la rend difficilement "jugeable". La Cour européenne des droits de l'homme l'a bien compris, estimant que si la matérialité des faits peut se prouver, les jugements de valeur ne se prêtent pas à une démonstration d'exactitude. En conséquence, quand les faits sur lesquels un journaliste fonde un jugement de valeur ne sont pas contestés, pas plus que sa bonne foi, condamner ce journaliste parce qu'il n'a pas pu établir la vérité de son assertion, exprimée dans ce jugement de valeur, porte atteinte à la liberté d'opinion et à la liberté de presse'.

C'est cette nature complexe de la vérité journalistique qui plaide pour une mise en commun des appréciations dans la polyrégulation. Comme les juges ont intérêt à s'ouvrir aux journalistes pour l'arbitrage des valeurs, les journalistes ont intérêt à s'ouvrir aux juges pour l'estimation de la vérité. Et pour ce faire, une instance partagée, avec des procédures communes, est toute indiquée.

\section{Des pratiques érodées}

Dernier argument pour une régulation croisée : les pratiques érodées des deux “corps". C'est notamment le point de vue de Christian Panier, ancien président du tribunal de première instance de Namur, pour qui l'effritement actuel des idéologies, la déliquescence de la norme du droit positif et l'entrechoquement constant des cultures judiciaire et médiatique se conjuguent pour engager la régulation des pratiques vers un système de normativité négociée.

1 Voir J. VELU, op. cit., p. 18. 
La mercantilisation croissante du métier de journaliste a transformé la presse de service en une presse de produit. Parallèlement, l'évolution de la société, avec la montée du bien-être, a modifié le travail du magistrat. Le justiciable "consumériste" ne pense plus qu'en rapport qualité-prix. Il a fait passer l'institution judiciaire du régime du droit-service au régime du droit-produit.

Les anciens consensus s'érodent, estime Christian Panier. Plus personne ne sait très bien où est sa place. On voit des journalistes se prendre pour des enquêteurs ou des magistrats, et des juges se muer en communicateurs, menant une partie de leurs investigations sur les plateaux de télévision. Quand les consensus et les déontologies s'effritent à ce point, il n'est plus possible de dire : c'est blanc ou c'est noir. Les points d'accord disparaissent, et la tension devient permanente. Il vaut alors mieux mettre tout le monde autour de la table pour gérer cette tension, et négocier la norme qui ne s'impose plus d'ellemême. A défaut de normes d'autorité, on entre dans un système de normes négociées, donc fluctuantes ${ }^{1}$.

Il faut, conclut Christian Panier, créer un lieu d'échange permanent " où on peut ajuster la norme en continu, avec les autres, et reconstruire une déontologie commune".

Antoine Garapon, au colloque du Sénat belge sur la justice et les médias, dressait un constat similaire ${ }^{2}$. Presse et justice ont perdu leurs marques, faisant au passage l'affaire des justiciables qui jouent désormais l'une contre l'autre au gré de leurs intérêts. Les médias sont tentés de faire justice eux-mêmes. Les juges sont guettés par l'illusion du contact direct avec l'opinion, en dehors des médiations éprouvées de la procédure. Or la société a besoin d'un équilibre entre ces acteurs.

Les médias ne se contentent plus de rapporter ce que fait la justice, de la critiquer au besoin, ce qui est leur rôle. Ils se mettent à copier les méthodes de justice et se targuent des mêmes qualités qu'un juge d'instruction : patience, minutie, ténacité. (...) Ils ne se contentent plus d'informer mais veulent intervenir directement dans le cours des événements. La télévision voudrait à la fois être metteur en scène et acteur. Or,

1 Interview de Christian Panier réalisée le 31 mars 1998 par l'auteur du présent article.

2 A. Garapon, "Justice et médias : une alchimie douteuse", in Coordonnées du colloque "Justice et médias", op. cit., p. 39-41. 
personne, ni aucun lieu ne peut prétendre incarner à soi seul l'espace public. Pas plus les institutions que les médias. Cet espace procède de l'équilibre -voire de la tension- entre un pouvoir institué et procédural, la justice, et un autre pouvoir, non institué et libre, la presse. Encore faut-il, précise Antoine Garapon, que chacun reste à sa place et qu'il soit sanctionné en cas de débordement.

La vertu d'une instance partagée de régulation devrait être, précisément, de permettre à ces deux pouvoirs -la justice et la presse- de retrouver leur place et de mieux sanctionner les débordements.

\section{Une régulation partagée}

Pour bien contrôler les relations presse-justice, pour bien évaluer les ingérences de la liberté d'informer dans le secret judiciaire et réciproquement, il faut donc être plusieurs, au même endroit, en confrontation permanente. C'est cette idée qui est à la base de notre proposition d" instance de régulation partagée ", valable pour le système belge comme pour tous les systèmes apparentés.

Cette instance s'inscrirait dans une économie de régulation désormais à trois pôles :

- le premier pôle resterait celui du contrôle déontologique interne des actes de presse, assuré actuellement par l'AGJPB en Belgique ;

- le deuxième pôle demeurerait celui du contrôle judiciaire externe, assuré par les parquets, cours et tribunaux ;

- le troisième pôle serait celui du contrôle mixte, assuré par notre instance, qui aurait rôle d'expert (à la fois conseil et arbitre) auprès des deux autres pôles pour les rapports spécifiques presse-justice.

La mission de cette instance serait donc de réguler, de manière théorique et pratique, la liberté de presse dans le domaine judiciaire.

Par régulation pratique, il faut entendre l'appréciation au cas par cas de l'usage fait de la liberté d'enquêter et d'informer, au regard des droits de la personne et du secret assurant le bon fonctionnement de la justice. Le même examen serait opéré quant à l'usage fait de ces droits et du secret judiciaire pour restreindre la liberté de presse. C'est l'aspect " prescriptif" de la régulation.

Par régulation théorique, il faut entendre la formulation d'avis et de recommandations dans le cadre général de la définition et de 
l'application du droit en matière de presse, comme de la déontologie journalistique en matière de justice. C'est l'aspect " normatif" de la régulation.

Les compétences de cette instance reposeraient sur des pouvoirs de sanction et d'orientation. Pour trancher les litiges qui lui seraient soumis ou formuler des recommandations, l'instance devrait entendre toutes les parties à la cause. Pour réprimer les mésusages de la liberté de presse ou du secret judiciaire, comme pour édicter des normes, elle s'instruirait des témoignages et de l'expérience de l'ensemble des acteurs, praticiens de presse et de justice.

C'est de cette manière que l'instance de régulation remplirait son rôle de conseil et d'arbitre. D'un côté, sur invitation ou d'initiative, elle donnerait des avis sur le cadre général et les dispositions particulières régissant les relations entre la presse et la justice. A cet effet, elle aurait vocation d' " expert-conseil " auprès d'interlocuteurs aussi variés que le ministre de la Justice et le collège des procureurs généraux, les parlementaires ou les responsables de l'AGJPB.

De l'autre côté, dans les cas singuliers de litige à trancher, elle serait amenée à circonscrire les pratiques irrégulières, à en établir les responsabilités et à proposer d'éventuelles sanctions. Elle aurait alors vocation d'" expert-arbitre " auprès des parquets, cours et tribunaux, comme auprès des instances déontologiques de l'AGJPB.

Plus concrètement, dans sa compétence générale de conseil (régulation normative), l'instance devrait notamment veiller à ce que les ingérences et restrictions de pratique -prévues ou existantes-en matière de liberté de presse relèvent bien des "mesures nécessaires" pour garantir l'autorité et l'impartialité du pouvoir judiciaire, comme le stipule l'article $10 \S 2$ de la Convention européenne des droits de l'homme. Ou, comme le demande la déclaration de Munich, que le secret des affaires publiques ou privées opposé au journaliste le soit en vertu de motifs "clairement exprimés ".

Tout aussi concrètement, dans sa compétence particulière d'arbitre (régulation prescriptive), cette instance devrait notamment se prononcer sur le respect, par la presse, de la présomption d'innocence des inculpés ; de la vie privée, de la réputation et de l'honorabilité des personnes en présence (inculpés, témoins, enquêteurs, magistrats, avocats... ) ; des droits de la défense (dont le droit à un procès équitable); de la bonne exécution des devoirs d'enquête ; de l'intégrité physique ou morale des personnes citées dans le dossier. Elle devrait également se prononcer sur le respect, par les autorités judi- 
ciaires, de l'égalité de traitement et d'accès à l'information de tous les journalistes.

En cas de non-respect, cette instance aurait à déterminer si les raisons à la "désobéissance" sont justifiables, voire légitimes, suivant un but supérieur à celui lié au respect des mesures : révélation d'une machination, d'un dysfonctionnement grave, d'une menace pour la sécurité des personnes ou l'ordre public, etc.

Notons que cette compétence d'expert-arbitre auprès des tribunaux matérialise 1'“ articulation harmonieuse " entre instance de régulation et juridictions traditionnelles prônée notamment par François Jongen. Pour ce dernier, une instance de ce type est mieux à même d'apprécier les droits, les devoirs et les conditions de fonctionnement d'un média, éclairant ainsi les décisions du juge de fond en la matière ou se chargeant elle-même du traitement des plaintes ${ }^{1}$.

La composition de cette instance, parce que partagée, devrait reposer sur la présence paritaire de membres issus du monde de la presse et du monde judiciaire. Cette parité est requise à deux titres : pour faire communiquer et se "confronter" ces deux mondes sur pied d'égalité, et pour éviter toute suspicion de mainmise d'un partenaire sur l'autre (la presse sur la justice, ou la justice sur la presse).

Outre la parité, la composition de cette instance exigerait également 1 " "autorité d'expérience " de ses membres. Les magistrats et les praticiens de la presse (journalistes et éditeurs) qui formeraient cette instance devraient connaître le sujet sur le bout des doigts. Leur expérience, leur intelligence du métier et leur qualité morale devraient être reconnues par leurs pairs pour que cette nouvelle instance fasse autorité.

Pour le reste, plusieurs questions restent ouvertes. Il faut éviter les conflits d'intérêt, lorsqu'un magistrat membre de cette instance devrait se prononcer sur un collègue de son propre ressort ou lorsqu'un éditeur (ou un journaliste) aurait à connaître d'un dossier impliquant son concurrent direct. On peut, par exemple, prévoir un nombre suffisant de suppléants appelés à " siéger" dans cette instance en remplacement de titulaires empêchés.

Quel mode de désignation pour ces membres? Il faut certainement qu'ils soient choisis par leurs pairs, mais on pourrait envisager

1 Fr. JONGEN, "Le rôle d'une autorité administrative indépendante dans le contrôle déontologique", in Prévention et réparation des préjudices causés par les médias, Bruxelles, Larcier, 1998, p. 48-49. 
qu'une partie d'entre eux soient désignés par une tierce autorité, le parlement par exemple (fédéral ou communautaire), sur listes multiples de candidats, de manière à renforcer le sérieux et la crédibilité de cette instance.

Le public y a-t-il sa place? Question délicate, pour deux raisons principales. La première tient au statut même du "public" dans ce genre d'enceintes. Le risque est grand que les représentants de ce public -dont le mode de désignation reste un casse-tête- soient davantage portés à surveiller leurs intérêts et ceux de leurs groupements (associations, lobbies, partis, syndicats...) qu'à faire entendre la voix des usagers ordinaires de la presse.

La seconde raison tient au statut particulier de cette instance de régulation. Amenée à entendre les parties à la cause en cas de litige, à jongler avec des principes de droit et de déontologie, à se prononcer sur des sanctions éventuelles de pratiques irrégulières, cette instance se prête mal à la présence d'un public " profane".

Mais la question du public reste posée. Un regard extérieur nonprofessionnel peut toujours offrir un intérêt à la régulation des pratiques, notamment pour éviter qu'en l'espèce, presse et justice s'arrangent, d'une manière ou d'une autre, sur le dos du public.

Le recours à des experts universitaires pourrait assurer cette présence externe, sans toutefois offrir le caractère "non initié " du grand public. Le présent article ne tranche pas. Il se contente de pointer les difficultés de procéduraliser la présence de tiers dans une telle instance.

Quant aux modalités de fonctionnement de cette instance, elles devraient être précisées dans un règlement d'ordre intérieur que le présent article n'aborde pas. Le principe resterait toutefois qu'en tant que conseil et arbitre, l'instance se ferait l'obligation d'entendre toutes les parties et de prendre en compte tous les arguments. Un rapport annuel d'activités contribuerait également à assurer la transparence de cette nouvelle instance. Quant à la prise de décision, la majorité serait préférée à l'unanimité, des "notes de minorité" pouvant être jointes au libellé final.

Quelques autres points méritent toutefois qu'on s'y attarde.

Le secret des délibérations semble inévitable pour garantir l'efficacité des travaux. Sans préjudice des lois et règlements en vigueur, on pourrait envisager que les magistrats et autres collaborateurs de justice (enquêteurs, personnel de greffes, avocats... ) puissent être déliés de leur secret professionnel devant cette instance, et que les 
journalistes puissent parler librement de leurs sources. Le point est délicat à régler. Cette levée des secrets professionnels impliquerait à tout le moins deux garanties formelles. La première est que les membres de cette instance de régulation soient à leur tour tenus au secret professionnel, et passibles des sanctions prévues par l'article 458 du code pénal belge en cas d'infraction (une disposition qui irait de soi pour les magistrats membres de l'instance, déjà professionnellement soumis au secret, mais qui pourrait poser problème aux autres membres, journalistes et éditeurs, non soumis au secret professionnel en dehors de l'instance). La seconde garantie formelle -si tant est qu'elle soit juridiquement possible- serait d'immuniser les comptes rendus d'auditions et de délibérations de cette instance de manière à ce que la justice pénale, après perquisition et saisie, ne puisse les utiliser dans ses procédures. Le problème est de taille.

La publicité des avis et sanctions semble tout aussi inévitable. Cette instance de régulation se doit de faire connaître ses décisions et leurs motivations. Mais deux questions se posent. Pourrait-elle d'initiative se saisir de cas d'irrégularités et les traiter en dehors de toute plainte ? La crainte est que cette instance se transforme en censeur omniprésent des pratiques médiatico-judiciaires. A l'inverse, si elle ne pouvait intervenir d'initiative, elle pourrait se voir confinée au seul rôle de conseil, sa fonction d'arbitrage tombant en désuétude. Il faut procéder à la balance des risques, mais lui refuser l'initiative pourrait handicaper son travail régulateur, d'autant que son pouvoir répressif reste limité. C'est la seconde question : doit-elle infliger ellemême les sanctions (du simple blâme aux mesures contraignantes plus lourdes) ou les recommander aux autorités compétentes ? La dernière solution semble la meilleure. Après avoir identifié les irrégularités et établi les responsabilités, cette instance de régulation se contenterait de recommander l'application d'une sanction aux instances disciplinaires concernées. A deux conditions toutefois : que ces instances (d'initiative ou à la requête des parties) puissent reprendre les procédures disciplinaires au début, pour confirmer le bien-fondé de la sanction, et que l'AGJPB s'aligne sur le personnel judiciaire (magistrats, services de police...) en se dotant d'instances disciplinaires compétentes, dont elle est aujourd'hui dépourvue.

Un degré d'appel serait également le bienvenu au sein de cette instance régulatrice. La simple publicité faite autour d'une recommandation de sanction (à l'adresse d'un journaliste, d'un éditeur ou d'un magistrat) constitue en soi une peine "morale" suffisante pour 
qu'elle puisse être frappée d'appel avant d'être effective. Il faudrait dès lors envisager la possibilité du recours et d'une "chambre" ad hoc au sein de cette instance de régulation. Ce n'est qu'une fois ce recours épuisé que la recommandation de sanction serait rendue publique.

\section{Du pain sur la planche}

Le travail ne manque pas pour renforcer conjointement la liberté de presse et la responsabilité sociale des médias. Qu'il s'agisse de la protection légale du secret des sources ou du repositionnement pénal du délit de presse, l'instance de régulation partagée pourrait utilement conseiller juge, déontologue et législateur.

Ainsi de la protection légale du secret des sources du journaliste, qui est une vieille revendication des professionnels du secteur. Ce secret des sources -à savoir la non-révélation par le journaliste de l'origine de ses informations- est à la base du réel pouvoir de la presse.

Grâce au secret, les journalistes peuvent entendre des témoins et recueillir des documents qui mettent en échec les autres pouvoirs quand ces derniers (la justice, les parlementaires, le gouvernement, mais aussi les partis, les syndicats, les lobbies et autres organisations) se retranchent derrière le silence ou le mensonge pour dissimuler des agissements coupables.

Le droit reconnu aux journalistes de taire l'origine de leurs informations est une des pierres angulaires de la liberté de presse, fondant le rôle de contrepoids démocratique des médias. Certains pays ont accepté de protéger légalement ce secret des sources journalistiques, comme la France, l'Allemagne, l'Espagne ou certains États des États-Unis.

La récente réforme du code français de procédure pénal (loi du 4 janvier 1993) dispose que " tout journaliste entendu comme témoin sur des informations recueillies dans l'exercice de son activité est libre de ne pas en révéler l'origine" (article 109-2)1.

Des résolutions adoptées par l'assemblée parlementaire du Conseil de l'Europe (résolution 1003 du ler juillet 1993) et le parlement de l'Union européenne (résolution du 18 janvier 1994) recom-

1 Voir H. PIGEAT, op. cit., p. 124. 
mandent aux États membres qui ne l'auraient pas encore fait d'assurer la confidentialité des sources d'information des journalistes. Motivation : cette confidentialité des sources est inhérente à la liberté de presse telle que définie par l'article 10 de la Convention européenne des droits de l'homme.

Une des premières tâches de l'instance de régulation, dans sa fonction de conseil, serait de proposer au législateur et aux juges des mesures (régime légal et normes d'appréciation) pour protéger le secret des sources en Belgique. Et dans sa fonction d'arbitre, elle serait amenée à évaluer, au profit des tribunaux ou des instances déontologiques de la presse, les contentieux liés au secret de ces sources.

Selon les cas, elle aurait à établir si le refus du journaliste de révéler ses sources est ou non justifié, si la suspicion d'une origine frauduleuse à ses informations est ou non fondée, ou si l'invocation des autorités judiciaires de circonstances exceptionnelles pour casser le secret est ou non légitime. Raison pour laquelle cette instance devrait elle-même travailler dans le secret le plus absolu de ses auditions et délibérations, permettant aux témoins et parties à la cause de s'expliquer en toute liberté.

D'autres mesures de protection du travail journalistique pourraient être étudiées par cette instance de régulation partagée.

Faut-il interdire la confiscation de matériel rédactionnel, les perquisitions dans les rédactions et au domicile des journalistes, les fouilles corporelles et les écoutes téléphoniques pour éviter que soit contournée la règle de la protection des sources ? Certains juristes estiment qu'aucune de ces interdictions ne s'impose car, pas plus qu'un cabinet d'avocat, d'un notaire ou d'un médecin, le bureau ou le domicile d'un journaliste ne peut devenir un asile contre les investigations de la justice pénale.

Toutefois, des dispositions particulières pourraient être prévues en cas de perquisition ou de saisie chez un journaliste ou dans un média. Comme en droit français, de tels devoirs judiciaires ne pourraient être menés que par un magistrat veillant à ce que l'exercice de la profession journalistique ne soit pas entravé. Il laisserait au journaliste une copie de chaque document saisi et empêcherait tout retard à la diffusion de l'information ${ }^{1}$.

1 Voir H. BosLY, "Les relations entre la justice et la presse. Aspects de droit pénal et de droit de la procédure pénale", in Justice et médias. Trois avis préliminaires à la demande du ministre de la Justice, partie A, Bruxelles, 1995, p. 17. 
En matière d'écoutes téléphoniques, un débat récent au parlement allemand a montré la situation singulière des journalistes, certaines formations politiques réclamant au bénéfice de la presse, comme à celui des avocats pénalistes, des mesures spécifiques dans le nouveau régime légal. En Allemagne, le droit au secret des sources est reconnu aux journalistes.

Perquisitions, saisies, écoutes, l'instance de régulation partagée pourrait préciser les contours de leur application aux journalistes et aux médias, de manière à garantir le juste exercice de la liberté de presse.

Par ailleurs, pour renforcer la responsabilité sociale de la presse, il faudrait revoir la qualification pénale de plusieurs infractions liées à la presse, comme le repositionnement du délit de presse.

Beaucoup estiment qu'en Belgique, les difficultés actuelles à poursuivre les délits de presse devant les jurys d'assises (procédures lourdes et coûteuses, engorgement des cours et parquets) génèrent des représailles devant d'autres juridictions. Insatisfaits des nonpoursuites, les victimes présumées du délit de presse, voire le ministère public, se rabattent sur d'autres infractions (comme le recel) ou sur le juge civil pour tenter d'ôter toute impunité aux journalistes et aux éditeurs.

Ces effets retors sont dénoncés depuis longtemps. L'actuel gouvernement belge a mis la correctionnalisation du délit de presse à son programme, et de nombreuses propositions de loi ont été déposées pour modifier les poursuites pénales en la matière. Dans cette redéfinition, l'instance de régulation partagée pourrait conseiller le législateur et à l'avenir, servir d'auditeur permanent du nouveau régime.

La même fonction pourrait lui être attribuée en matière de recel et de viol du secret judiciaire, en regard desquels le statut juridique des journalistes belges doit être précisé. D'un côté, le déblocage pénal attendu pour le délit de presse devrait clarifier les poursuites, les "vengeances" par voie de recel ou de complicité de viol du secret professionnel devant tomber d'elles-mêmes. De l'autre, la protection légale du secret des sources, une fois attribuée aux journalistes, forcera à revoir les qualifications et procédures d'enquête judiciaires en matière de recel et de viol du secret à charge de la presse.

Parmi les chantiers possibles pour cette instance de régulation, on peut encore penser au droit de réponse (le rendre applicable, par exemple, dans un délai de plusieurs mois après toute décision de non 
lieu ou d'acquittement), à l'insertion forcée de communiqués judiciaires dans la presse ou à l'interdiction de publier certains noms.

On peut enfin signaler deux champs d'intervention qui seront parmi les plus malaisés à circonscrire pour cette instance. Il y a le rôle joué par le juge des référés en matière de presse. Pour réduire les risques de censure, et accroître la sécurité juridique, le législateur devrait préciser les modalités de saisie en urgence et les cas où une interdiction de publier se justifie.

Il y a également la responsabilité civile des journalistes en matière de quasi-délits (articles 1382 et suivants du code civil belge). On peut s'attendre, à l'avenir, à une diminution des recours devant le juge civil si tant la poursuite pénale des délits de presse que la régulation prescriptive des journalistes sont rendues effectives. Mais il sera toujours loisible au justiciable d'invoquer un lien de cause à effet entre un article de presse et le moindre dommage subi, et d'en demander réparation.

Comme pour le juge des référés, l'intervention du juge des quasidélits devra être exempte de toute velléité " punitive " à l'adresse de la presse. Le législateur doit, ici aussi, préciser les contours de responsabilité civile des médias, l'objectif étant d'empêcher la transformation de la juridiction civile en arme de dissuasion. Le tri doit être plus facilement opéré, à l'avenir, entre les "vrais" recours en dédommagement pour préjudice établi et les "fausses" actions dissimulant des tentatives de déstabilisation d'un journaliste ou d'un média.

Dans le même ordre d'idées, l'application de la responsabilité en cascade en matière civile devrait être mieux délimitée. Jusqu'il y a peu, cette responsabilité en cascade, sur base de l'article 25 alinéa 2 de la Constitution belge ("Lorsque l'auteur est connu et domicilié en Belgique, l'éditeur, l'imprimeur ou le distributeur ne peut être poursuivi"), ne s'appliquait qu'en matière pénale. La plupart des juridictions civiles ignoraient ce principe, considérant que la publication d'un article pouvait aussi engager la responsabilité d'un rédacteur en chef ou d'un éditeur quand un manque de contrôle ou de surveillance de leur part sur le travail du journaliste avait entraîné un préjudice pour autrui.

Dans son arrêt du 31 mai 1996, la Cour de cassation belge en a jugé autrement, estimant que le civil devait s'aligner sur le pénal et s'arrêter à la seule responsabilité du journaliste quand celui-ci était connu : rédacteur en chef et éditeur ne pouvaient être solidairement entraînés dans le dommage causé. 
Si cet arrêt voulait mettre fin à une vieille controverse, il n'a pas réussi à apaiser les esprits dans la mesure où il a trop peu pris en considération le lien de subordination existant entre les journalistes -salariés ou indépendants- et les décideurs rédactionnels ${ }^{1}$. L'article publié, même s'il n'a pas été commandé par la hiérarchie du média, est proposé au public sous l'autorité éditoriale du rédacteur en chef ou d'un représentant de la direction. Ces derniers ont la responsabilité finale de la publication. En matière civile en tout cas, ils peuvent difficilement être exemptés de toute erreur. A nouveau, un avis mûri de l'instance de régulation serait le bienvenu.

Pour terminer, signalons qu'en plus des mesures légales définissant l'exercice combiné de la liberté de presse et des droits concurrents, l'instance de régulation partagée pourrait formuler des recommandations sur tout ce qui a trait aux rapports ordinaires entre l'institution judiciaire et les médias.

Figurent en bonne place les communications du parquet à la presse, que réglementent plusieurs circulaires ministérielles et que la loi belge du 5 mars 1998 vient d'officialiser. Les principes sont connus. Il faut permettre au public de savoir tout en préservant l'efficacité des enquêtes et le droit des personnes (présomption d'innocence, honorabilité, droits de la défense...).

C'est l'application des principes, au cas par cas, qui peut poser problème. Quand l'équilibre est-il rompu entre le droit du public à l'information et le droit de la justice au secret? Dans une même situation, l'attitude des parquets peut considérablement varier, du porte-parole volubile, confiant le tri des informations à la sagacité des journalistes, au magistrat crispé à la moindre vue d'une carte de presse. Les conseils de l'instance de régulation pourraient contribuer à harmoniser les pratiques.

Le recours aux médias électroniques (émissions de télévision, réseau internet...) pour aider à l'élucidation d'affaires criminelles tend à se généraliser. Le public pouvant contribuer à faire sortir des enquêtes de l'impasse, de tels recours sont utiles mais la question des limites se pose.

Toute investigation qui dure ouvre-t-elle droit aux plateaux de télévision ? Faut-il s'en tenir aux faits graves ? L'accord des victimes ou des familles est-elle un préalable ? L'instance de régulation parta-

I Voir E. Montero, "La responsabilité civile des médias", in Prévention et réparation des préjudices causés par les médias, op. cit.p. 96-100. 
gée pourrait avantageusement abriter les débats d'orientation en la matière.

Mentionnons encore la formation continue des magistrats et des journalistes. Les parquets manquent parfois d'expérience en matière de presse. La logique et les processus du "dispositif médiatique" échappent encore à certains porte-parole. Dans le même temps, tous les journalistes ne brillent pas par leurs connaissances juridiques. Les arcanes de la procédure ou la subtilité d'une décision en découragent plus d'un. Les médias, en général pressés, s'accommodent mal des prudences de l'institution judiciaire.

Dans sa fonction de conseil, l'instance de régulation pourrait proposer des standards de formation ou de remise à niveau pour juges et journalistes. L'objectif serait de favoriser un climat de confiance entre la presse et l'appareil judiciaire, afin d'accroître chez chacun la connaissance de l'autre. Une collaboration bien comprise ne peut que renforcer le service rendu aux justiciables et à l'opinion publique.

\section{En conclusion}

Aucune institution ne peut fonctionner sans la bonne volonté des acteurs. L'instance de régulation partagée, proposée pour contrôler les usages de la liberté de presse et du secret judiciaire, n'y échappera pas. Elle ne pourra réussir qu'en comptant sur l'engagement résolu et bienveillant des représentants des deux mondes.

Juges et journalistes doivent pour cela dépasser les antagonismes. Le présent article a voulu montrer en quoi ces derniers étaient contreproductifs en matière de régulation. Ils font se cabrer les parties en cause sans conduire à la résolution des conflits. Les médias font de la déontologie un rempart. La justice use de tous les béliers que lui confère le droit. Et le siège s'éternise, sans réel vainqueur.

Les réflexes de citadelle assiégée, en réalité, ne servent personne. Chaque régulateur a un intérêt légitime à faire valoir. Si la liberté de presse s'affaiblit vite devant des limites trop rigoureuses, elle devient par contre tyrannique en l'absence de toute contrainte. Il faut faire la balance des droits et des ingérences respectives, et cette tâche est mieux réalisée à plusieurs, en un même lieu, en concertation permanente, que chacun de son côté, en bagarres répétées.

Le succès de cette régulation partagée ne sera toutefois pas assuré parce qu'elle formerait le juste milieu entre toutes les revendi- 
cations, sorte de moyenne arithmétique des bonnes volontés. Il ne suffira pas d'asseoir à la même table praticiens des médias et de la justice, même bien disposés, pour rendre effectif le contrôle combiné de la liberté de presse et du secret judiciaire. La mixité bienveillante est nécessaire mais insuffisante. Il faut encore reconnaître à l'autre la validité de son jugement.

Ce sera sans doute le défi le plus difficile à relever pour cette instance. Juges et journalistes se parlent déjà. Ils se rencontrent lors de colloques. Mais quelle légitimité accordent-ils au point de vue de l'autre ? L'impression du " cause toujours " prédomine. Quoi qu'en pense la presse, la justice s'estime la seule garante de l'équilibre des droits. Quoi qu'en disent les juges, les journalistes s'estiment les vrais défenseurs de la liberté de presse et de l'opinion publique.

Dépasser la concurrence et l'esprit d'exclusive sera l'autre condition, indispensable, à la régulation partagée. Si juges et journalistes ne se reconnaissent pas mutuellement la compétence ou l'autorité " naturelles" pour juger des usages de la liberté de presse et du secret judiciaire, les décisions de la nouvelle instance seront sans effet.

Après avoir consenti à des exercices partagés de régulation, justice et presse en reviendront, discrètement, à leurs vieilles habitudes. Chacun, sur le terrain, ne fera confiance qu' aux hommes de son camp. La nouvelle instance, ce sera pour la forme. Le véritable contrôle restera entre les mains de l'appareil judiciaire, pour les uns, des instances journalistiques, pour les autres.

Juges et journalistes poursuivent pourtant le même but, celui de servir le public, par la manifestation de la vérité. C'est d'ailleurs ensemble, comme le dit Garapon, par leur équilibre, que la justice -pouvoir institué et procédural- et la presse-pouvoir non institué et libre- incarnent l'espace public. Divisés et rivaux, ils le morcellent.

Cette incarnation de l'espace public, ce service du citoyen-justiciable sont au fondement de toute régulation, de la presse comme de la justice. Même sans contrôle combiné, juges et journalistes doivent se le rappeler. Une instance partagée, à cet égard, aurait au moins le bénéfice de consolider les mémoires ${ }^{1}$.

1 Cet article est un résumé du mémoire de DEA présenté en septembre 1998 au Département de communication de l'UCL. 